\section{Discovery of Tumor Necrosis Factor (TNF) and Identification of the Potential of Anti-TNF Antibodies in Dr. Cerami's Laboratory}

\author{
Masanobu Kawakami, ${ }^{1,2}$ \\ ${ }^{1}$ Nerima Hikarigaoka Hospital, Tokyo, Japan; and ${ }^{2}$ Saitama Medical Center, Jichi \\ Medical University, Saitama, Japan
}

Online address: http://www.molmed.org doi: $10.2119 / \mathrm{molmed} .2014 .00177$
The beginning of my exciting journey for this work began in 1978 when I was a postdoctoral fellow at Columbia University and attended a lecture at Grand Rounds by a young professor from the Rockefeller University named Anthony Cerami. His topic was the identification of a new test for measuring glucose control in diabetes patients over a 1- to 2month period. He and Ronald Koenig, a graduate student in his laboratory, had discovered that glucose could slowly react with hemoglobin in red cells of mice and humans to form a minor hemoglobin called hemoglobin $\mathrm{A}_{1 \mathrm{c}}\left(\mathrm{Hb} \mathrm{A}_{1 \mathrm{c}}\right)$. They showed that the amount of $\mathrm{Hb} \mathrm{A}_{1 \mathrm{c}}$ compared with the parent molecule $\mathrm{Hb}$ A could be used as an integrator of blood glucose over the previous 1-2 months. This test has gone on to be the major measure of diabetic control as well as diagnostic for identifying patients with diabetes throughout the world. I was im- pressed with the rational way they went about proving the validity of the test, which could be used to understand the importance of glucose control in preventing diabetes complications. Large clinical trials in type 1 and type 2 diabetes proved without a doubt that good glucose control measured by $\mathrm{Hb} \mathrm{A_{1c }}$ could prevent diabetes complications. After the lecture, he invited the audience to contact him if someone wanted to join him on a new research project.

Through the good graces of Shigeru Sassa, I was formally introduced to Dr. Cerami and his clinical colleague Dr. Charles Peterson. One year later, I was finally able to move from the Hudson River at 168th Street to the East River and 68th Street and join the Laboratory of Medical Biochemistry. I quickly discovered that Dr. Cerami was interested in fields other than diabetes, including malaria, trypanosomiasis and iron over-
Address correspondence to Masanobu Kawakami, Nerima Hikarigaoka Hospital, 2-1 1-1 Hikarigaoka, Nerima-ku, Tokyo 179-0072, Japan. Phone: +81-3-3979-361 1; Fax: +81-3-39793787; E-mail: nobukawa@omiya.jichi.ac.jp.

Submitted September 9, 2014; Accepted for publication September 9, 2014; Published Online (www.molmed.org) December 16, 2014.

\section{The Feinstein Institute} for Medical Research Empowering Imagination. Pioneering Discovery.

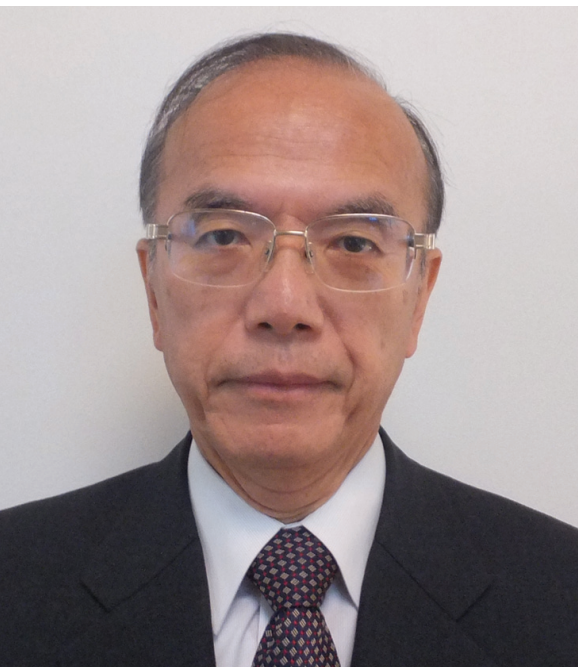

load due to thalassemia, to name a few. I also observed that he would spend considerable time discussing in detail these projects with all members of the lab, including student and postdoctoral fellows. He always wanted to know "what was new." I found this overwhelming and very different from the Japanese hierarchal culture from which I came. I was also surprised to see how many projects were ongoing in completely different disciplines. It took me years to understand that they were all related in his mind and that his interest was focused on entire systems instead of single organs or traditional isolated disciplines.

When I first started working in the laboratory, I worked on lipoprotein metabolism in diabetes. The idea was that perhaps lipoproteins were nonenzymatically glycosylated by glucose in diabetes, which would interfere with uptake by the LDL receptor that had recently been discovered by Brown and Goldstein. One day, Carol Rouzer, an MD/PhD student in the lab, asked for my help in identifying what lipoproteins were responsible for the white, opaque lipemic sera that she found in the blood of rabbits that she had infected with trypanosomes. The origin of this project was to understand why cattle infected with this parasite developed cachexia, which was associated with anemia, loss of appetite and severe 
loss of weight (all hallmarks of cachexia). Carol's experiments in rabbits revealed that the anemia in the rabbits was related to an increased rate of red cell removal from the blood and a decreased rate of production of new red cells in the infected animals - a clue to understanding the anemia associated with cachexia. During the course of her studies, she noticed that, after infection, lipoproteins were accumulating in the blood to such a degree that it was noticeable to the eye. At this time, Carol decided that this was too difficult and risky for a $\mathrm{PhD}$ thesis and decided to move to another lab. The next thing I knew, Dr. Cerami put me in charge of the project, since he thought that at least I knew something about lipoproteins.

I decided that the best thing to do was to review the literature of metabolic derangements that occur in different diseases. It is hard to imagine today how difficult it was to collect papers on a specific topic at that time without the aid of computers. I spent many hours in the Rockefeller library collecting papers dealing with metabolic derangements in a variety of exogenous and endogenous insults, including infections, endotoxemia, cancer and allergic diseases. As was his custom, Dr. Cerami would read the papers that I found and discuss the contents in detail. It was clear to us from this reading that there was a commonality in the response of the mammal to invasion, which was the onset of hyperlipidemia. We hypothesized that there was a mediator that was being induced that caused the loss of the enzyme lipoprotein lipase in the capillaries of the body. The loss of this enzyme is critical for the cleavage of triglycerides in lipoproteins in the blood to glycerol and free fatty acids, which could then be taken up by endothelial cells and transported out of the bloodstream to muscle and fat cells, where they could be metabolized.

The question was how could we examine this idea in experimental animals. We quickly ruled out studying any further rabbits infected with trypanosomes and chose a mouse model of endotoxemia.
Endotoxin, which is prepared from the cell wall of bacteria, causes profound metabolic disturbances and death when injected into animals, including humans. Of interest, we read a paper that noted there were two strains of mice that were closely related. One was sensitive to endotoxin $\mathrm{C} 3 \mathrm{H} / \mathrm{He} \mathrm{N}$ (causing the death of the animal) and the other, $\mathrm{C} 3 \mathrm{H} / \mathrm{He} \mathrm{J}$, was not killed. Our hypothesis was that, if a "new mediator" was made in response to endotoxin, the injection of endotoxin into the $\mathrm{N}$ mouse would lead to the production of a "mediator" that could suppress lipoprotein lipase in the J mouse. In preliminary experiments, we showed that endotoxin injected into the J mouse did not decrease lipoprotein lipase. I was thrilled in one midnight session when the scintillation counter started to print out very low counts, which meant that a new mediator was being made by the $\mathrm{C} 3 \mathrm{H} / \mathrm{He} \mathrm{N}$ that could suppress lipoprotein lipase in the J mouse. This was a happy conclusion to many days of hard work. With this experiment, we could prove that endotoxin induced the production of a mediator that could suppress lipoprotein lipase. As in most successful experiments, many new questions were brought forward that demanded answers. How could we easily measure the amount of the mediator? How could we produce significant amounts of this mediator? What was this mediator? What cells produced it? What other biological activities were associated with it? How could we inhibit it?

The next issue that I addressed was the source of the mediator. Dr. Cerami suggested that macrophages might be a good place to start, since Dr. Zanvil Cohn's lab at the Rockefeller University had extensive experience in studying this cell. This theory turned out to be correct. The incubation of peritoneal macrophages from $\mathrm{C} 3 \mathrm{H} / \mathrm{He} \mathrm{N}$ mice with endotoxin produced a mediator that could suppress lipoprotein lipase in $\mathrm{C} 3 \mathrm{H} / \mathrm{HeJ}$ mice. This observation was a big step forward for our project, since we could produce the mediator by growing peritoneal macrophages in vitro and induce the mediator by adding endotoxin. Now we had a source for our mediator. Now we needed a simpler bioassay for the mediator.

One day, Dr. Cerami informed me that I was going to Johns Hopkins Medical School to work in Dr. Dan Lane's lab for a few weeks, which turned into months. Dr. Lane had been working on a special fibroblast cell line, 3T3-L1, which could be converted in vitro into adipocytes. Working with Phil Pekala, a junior member of Dr. Lane's lab, we established the presence of the mediator and its other effects on lipid metabolism in adipocytes. We also saw a significant induction of insulin resistance when the mediator was added to the cells. This result correlated with the clinical observation that, after infection, patients would have insulin resistance. I have good memories of the months that I worked on adipocytes during the week in Baltimore and worked on macrophages on weekends in New York.

During this time, I also collaborated with Dr. Sassa at the Rockefeller University. We were able to show that the bioactive material that we induced in macrophages had the ability of inhibiting red cell production in vitro. This finding was a pleasing result, since it was the phenomenon of decreased red cell production in rabbits infected with trypanosomes that gave the initial clue of hyperlipidemia that allowed us to go forward.

The development of the 3T3-L1 cell assay was an excellent bioassay for the mediator and an important step in our work. We quickly were able to show that the mediator was a protein with a molecular weight of about $70 \mathrm{kDa}$. We decided to call this mediator "cachectin" because of its presumed role (and subsequently demonstrating in mice) to play a major role in the phenomenon of cachexia. We subsequently wrote a U.S. patent describing our work on the characteristics of cachectin and the roles that it played in many pathological conditions. We proposed that neutralization of cachectin by monoclonal antibodies 
would be useful in the treatment of a number of human conditions, including rheumatoid arthritis. This patent was approved by the U.S. Patent and Trademark Office and became the cornerstone patent for all antibody-related patents that deal with the the neutralization of cachectin (also, see below on tumor necrosis factor [TNF]).

At this point in the story, I decided to return to my home in Japan. Dr. Cerami tried hard to encourage me to stay and continue the project to its completion, since much work needed to be done. I assured him that I would find a Japanese postdoctoral fellow as a comparable replacement.

After I returned to Japan, Dr. Cerami's lab was able to clone and sequence the gene for cachectin. Surprisingly, cachectin was found to be the same as another cytokine that had been cloned a few months earlier-TNF. When I was still working in New York, I had arranged an exchange of a preparation of cachectin for a preparation of TNF with members of Dr. Lloyd Old's laboratory at Sloan Kettering, who were pioneers in this area and across the street. In our 3T3-L1 assay, their primitive "TNF" had no cachectin activity, and our cachectin had no activity in their TNF bioassay. Why these experiments did not work is not obvious. It certainly would have saved a lot of time and effort.

After a hiatus of many years, I collaborated again with Dr. Cerami on another project that had origins from our initial cachectin project. Many years ago, Dr. Cerami proposed to me that there must be a way to shut off TNF production. His laboratory searched for a number of years for this natural anti-TNF without success, although his laboratory did discover a number of other proinflammatory cytokines. In the late 1990s, they discovered that locally produced nonglycosylated erythropoietin could shut off the production of TNF and other proinflammatory cytokines. Animal studies showed remarkable effects of erythropoietin in many animal models, for example, myocardial infarction and head trauma. Attempts to use recombinant human erythropoietin (rhEPO) in patients revealed positive effects, but that rhEPO was also promoting coagulation in the patients. Obviously, this effect prohibits the use of rhEPO for tissue protection. Typical for Dr. Cerami, they saw this as an opportunity to develop new agents that were antiinflammatory but were not hematopoietic or thrombopoietic. One of these molecules, ARA290, is an 11-amino acid peptide that is antiinflammatory and tissue protective but is not erythropoietic or thrombopoietic. With my colleagues in Japan, we have demonstrated the unique ability of ARA290 to decrease pathology in several animal models of cardiomyopathy. We anxiously await clinical studies with ARA290.

\section{DISCLOSURE}

M Kawakami is one of two inventors of anti-TNF antibody-related patents of the Rockefeller University and a science advisor and a stockholder of Araim Pharmaceuticals, which is developing the clinical application of ARA290. 